\title{
Magnetic resonance imaging for the assessment of myocardial viability
}

\author{
Viktor Peršić* \\ Thalassotherapia Opatija \\ - Clinic for treatment, \\ rehabilitation and prevention \\ of cardiovascular disease, \\ Opatija, Croatia
}

KEYWORDS: magnetic resonance of the heart, viability, myocardial infarction. CITATION: Cardiol Croat. 2015;10(3-4):88. | DOI: http://dx.doi.org/10.15836/ccar.2015.88 ORCID: Viktor Peršić, http://orcid.org/0000-0003-4473-5431

*ADDRESS FOR CORRESPONDENCE: Viktor Peršić, Thalassotherapia Opatija, M. Tita 188/1, HR-51410 Opatija, Croatia. Phone: +385-51-202-728. E-mail: viktor.persic@ri.t-com.hr

|IIIIIIIIIIIIIIIIIIIIIIIIIIIIIIIIIIIIIIIIIIIIIIIIIIIIIIIIIIIIIIIIIIIIIIIIIIIIIIIIIIIIIIIIIIIIIIIIIIIIIIIIIIIIIIIII

Coronary artery disease remains the leading cause of death in Europe and the leading cause of heart failure. In the management of therapeutic procedures in patients with heart failure caused by coronary heart disease is crucial assessment of myocardial viability, an important procedure that needs to be addressed when patients with dysfunctional myocardium are considered to be revascularised. The benefit of revascularization is greater if the supply area of the affected coronary blood vessels has enough viable myocardium whose revascularization achieve a functional benefit and significant impact on the prognosis and survival of patients.

Assessment of myocardial viability can be detected by nuclear techniques (PET, SPECT), stress echocardiography and magnetic resonance imaging (MRI). In the latter helps a combination of several MRI protocols: Cine MRI, perfusion MRI, delayed contrast enhancement. MRI of the heart is not just a tool, it makes a number of integrated tools in a unified search.The presentation will show the potential of MRI in the managing diagnostic and therapeutic approach in patients with known coronary heart disease.
RECEIVED:

April 15, 2015

ACCEPTED:

April 20, 2015

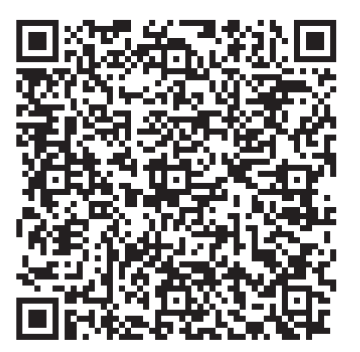

